\title{
Photon strength functions and the Oslo method
}

\author{
M. Guttormsen*', A.C. Larsen, J. Rekstad, S. Siem, N.U.H. Syed \\ Department of Physics, University of Oslo, Norway \\ E-mail: magne.guttormsen@fys.uio.no
}

\section{A. Schiller, A. Voinov}

Department of Physics and Astronomy, Ohio University, Athens, OH 45701, USA

\begin{abstract}
The essence of the Oslo method is explained and blind tests of the validity of the method are demonstrated. Furthermore, we focus on experimentally extracted photon strength functions (PSF) from various mass regions. The scissors mode pygmy resonance observed in the rare earth region is discussed and compared with other experiments. Of particular interest is the strong enhancement of low energy $\gamma$-rays observed for nuclei lighter than mass number $\mathrm{A} \sim 100$. This so-called $\gamma$ upbend of the PSF has still no theoretical explanation.
\end{abstract}

Workshop on Photon Strength Functions and Related Topics

June 17-20 2007

Prague, Czech Republic

\footnotetext{
${ }^{*}$ Speaker.

${ }^{\dagger}$ Financial support from the Norwegian Research Council (NFR) is acknowledged.
} 


\section{The Oslo method}

As in life, our loved ones carry several names. This community is in love with the $\gamma$ decay in continuum, called e.g. $\gamma$-ray strength function (GSF), radiative strength function (RSF) or photon strength function (PSF). As a gesture to the Prague group, we will use the later notation in this work.

The Oslo nuclear physics group has developed a method to extract simultaneously photon strength function (PSF) and nuclear level density (NLD) from particle- $\gamma$ coincidences. The experiments are performed with light-particle inelastic or transfer reactions. The charged ejectile is used to tag excitation energies for each $\gamma$-ray spectrum from the ground state up to the neutron (or proton) binding energy. The particle- $\gamma$ coincidences are measured with the highly efficient CACTUS detector set-up at the Oslo Cyclotron Laboratory.

The experimental extraction procedure and the assumptions made are described in Ref. [1] and references therein. For each initial excitation energy $E$, determined from the ejectile energy and reaction $Q$ value, $\gamma$-ray spectra are recorded. Then, the spectra are unfolded using the known $\gamma$-ray response function of the CACTUS array [2]. These unfolded spectra are the basis for making the first-generation (or primary) $\gamma$-ray matrix [3].

An important ingredient in the first-generation method is the knowledge of the number of $\gamma$ rays per cascade, called the $\gamma$-ray multiplicity $M_{\gamma}$. This quantity is needed to normalize each $\gamma$ spectrum to each other by multiplying with $M_{\gamma} / N_{c}$, where $N_{c}$ is the intensity of the particle $-\gamma$ coincidence spectrum. In this way the area of each $\gamma$ spectrum corresponds to as if we have the same reaction cross-section for all excitation energies. Furthermore, in the extraction of the primary $\gamma$ spectrum $P$, we subtract the weighted sum $B$ of underlying $\gamma$-spectra from the total spectrum $T$. Here, we have to assure that the multiplicities of the three spectra are correct, namely $M(P)=1$, $M(T)=M_{\gamma}$ and $M(B)=M_{\gamma}-1$.

The average $\gamma$-ray energy of the total $\gamma$-ray spectra at each excitation energy $E$ is expressed by

$$
\left\langle E_{\gamma}(E)\right\rangle=\frac{N E}{\sum_{n=1}^{N} M_{n}(E)},
$$

where $N$ is the number of $\gamma$-cascades from excitation energy $E$, and $M_{n}$ is the $\gamma$-ray multiplicity for the $n$th cascade. Thus, the average $\gamma$-ray multiplicity of the $N$ cascades is given by

$$
M_{\gamma}=\frac{1}{N} \sum_{n=1}^{N} M_{n}=\frac{E}{\left\langle E_{\gamma}\right\rangle} .
$$

For nuclei where the lowest ground band transitions have energies below the detection limits for the $\mathrm{NaI}$ detectors, we adopt the statistical $\gamma$-ray multiplicity given by

$$
M_{\gamma}^{\text {stat }}=\frac{E-E_{\text {entry }}}{\left\langle E_{\gamma}\right\rangle_{>E_{0}}},
$$

where typically $E_{\text {entry }}=0.5 \mathrm{MeV}$ and $E_{0}=0.3 \mathrm{MeV}$ for well deformed rare earth nuclei.

Figure 1 shows the $\gamma$-ray multiplicity versus excitation energy in molybdenum from the $\left({ }^{3} \mathrm{He}, \alpha\right)$ and $\left({ }^{3} \mathrm{He},{ }^{3} \mathrm{He}^{\prime}\right)$ reactions [4]. In spite of the different reaction mechanisms, the two reactions give 


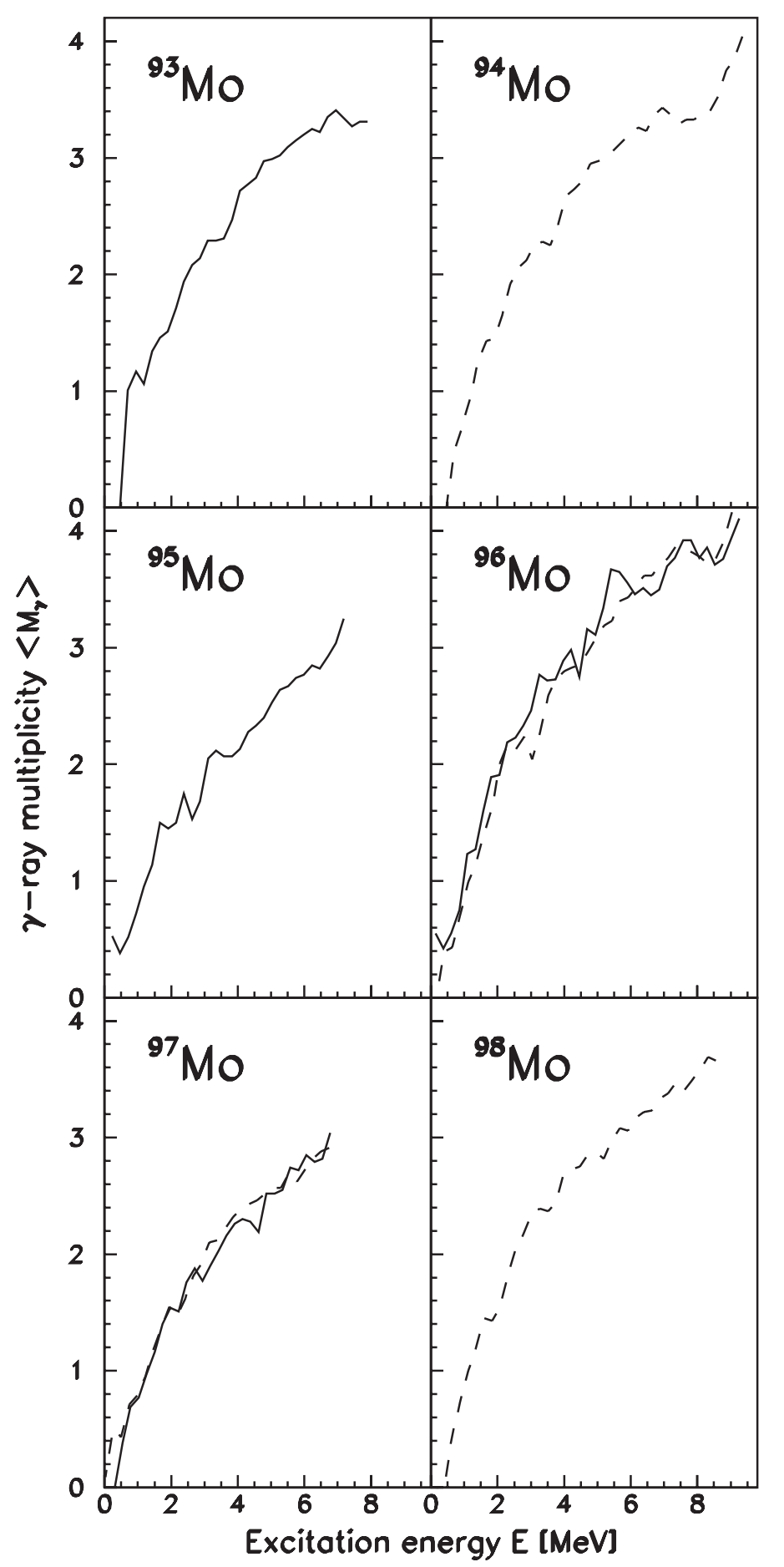

Figure 1: Gamma-ray multiplicity versus excitation energy. The individual spectra are labeled by the product nuclei. Solid and dashed lines represent $\left({ }^{3} \mathrm{He}, \alpha\right)$ and $\left({ }^{3} \mathrm{He},{ }^{3} \mathrm{He}^{\prime}\right)$ reactions, respectively. 


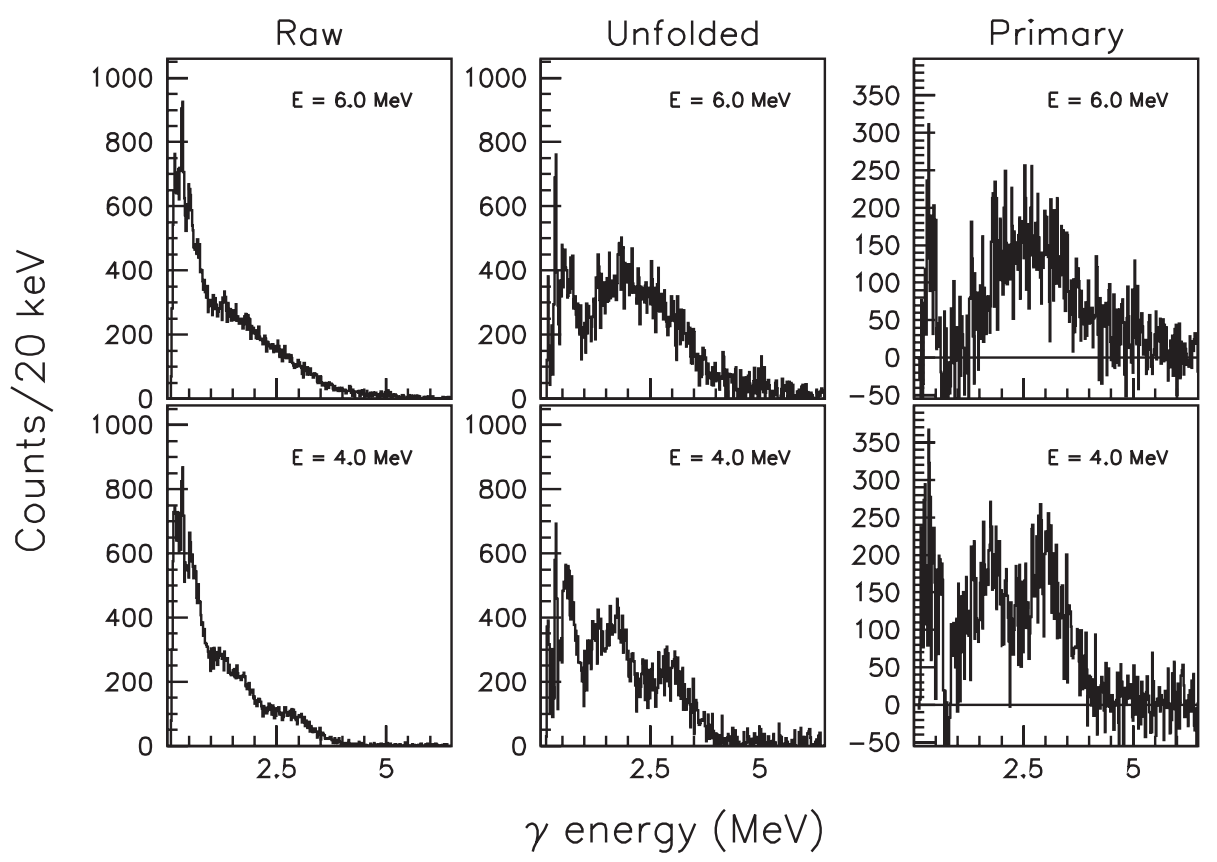

Figure 2: Gamma-ray spectra at $E=4$ and $6 \mathrm{MeV}$ of excitation energy for ${ }^{166} \mathrm{Er}$. From left to right are shown raw $\gamma$-ray spectra, unfolded $\gamma$-ray spectra, and first-generation $\gamma$-ray spectra.

similar results. In particular, the multiplicities (solid and dashed lines) of ${ }^{96} \mathrm{Mo}$ and ${ }^{97} \mathrm{Mo}$ are equal within their error bars, which give support to the applicability of the Oslo method for both reactions.

Figure 2 shows spectra for ${ }^{166} \mathrm{Er}$ and ${ }^{167} \mathrm{Er}$ at different steps of the Oslo method [5]. The directly recorded $\gamma$ spectra, the so-called raw spectra, from two excitation energies are shown in the left panels. The figure also includes spectra unfolded with the NaI response function (middle panels) and the final first-generation spectra (right panels).

The next step of the Oslo method is to factorize the first-generation $\gamma$ matrix $P\left(E, E_{\gamma}\right)$ according to the Brink-Axel hypothesis [6, 7] by

$$
P\left(E, E_{\gamma}\right) \propto \rho\left(E-E_{\gamma}\right) \mathscr{T}\left(E_{\gamma}\right)
$$

Here, $\rho$ is the level density and $\mathscr{T}$ is the photon transmission coefficient.

The $\rho$ and $\mathscr{T}$ functions can be determined by an iterative procedure [1] through the adjustment of each data point of these two functions until a global $\chi^{2}$ minimum of the fit to the experimental $P$ matrix is reached. It has been shown [1] that if one solution for the multiplicative functions $\rho$ and $\mathscr{T}$ is known, one may construct an infinite number of other functions, which give identical fits to $P$ by

$$
\begin{aligned}
\tilde{\rho}\left(E-E_{\gamma}\right) & =A \exp \left[\alpha\left(E-E_{\gamma}\right)\right] \rho\left(E-E_{\gamma}\right) \\
\tilde{\mathscr{T}}\left(E_{\gamma}\right) & =B \exp \left(\alpha E_{\gamma}\right) \mathscr{T}\left(E_{\gamma}\right)
\end{aligned}
$$

Consequently, neither the slope $(\alpha)$ nor the absolute values of the two functions ( $A$ and $B$ ) can be obtained through the fitting procedure. 


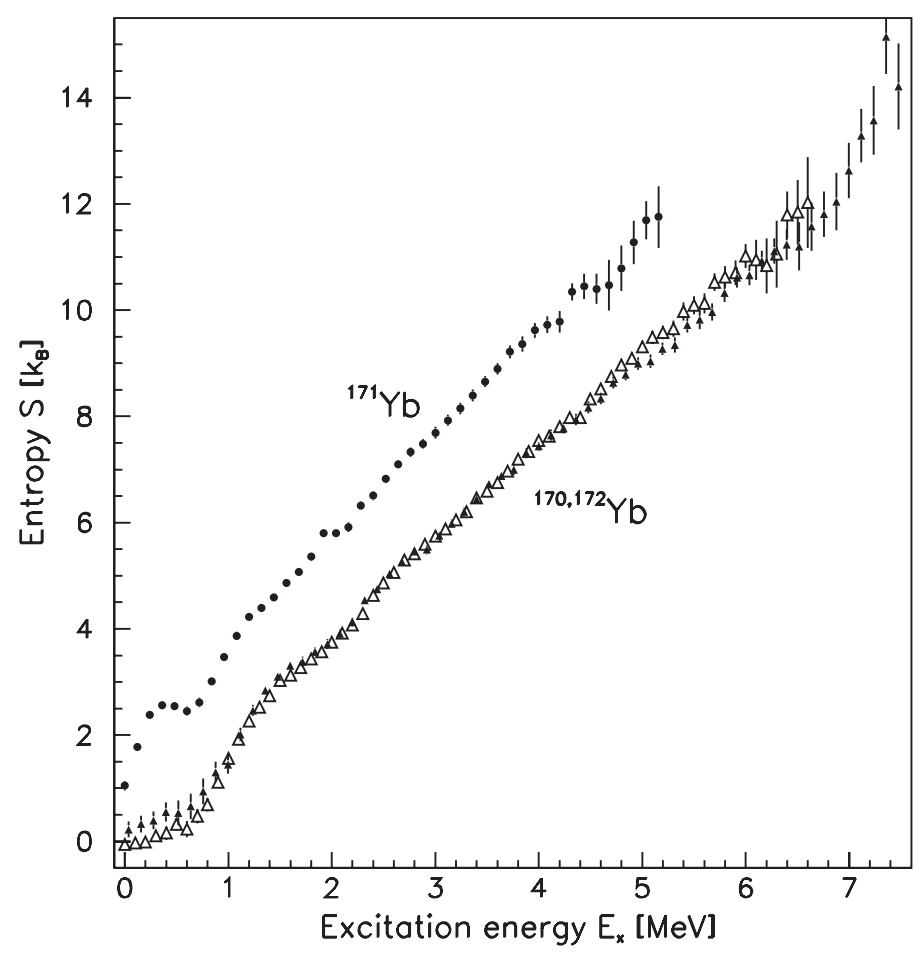

Figure 3: Deduced entropies for ${ }^{170,171,172} \mathrm{Yb}$ using the $\left({ }^{3} \mathrm{He}, \alpha\right)$ reaction. The full circles correspond to ${ }^{171} \mathrm{Yb}$. The full and open triangles correspond to ${ }^{170} \mathrm{Yb}$ and ${ }^{172} \mathrm{Yb}$, respectively.

The parameters $A$ and $\alpha$ can be determined by normalizing the level density to the number of known discrete levels at low excitation energy and to the level density estimated from neutronresonance spacing data at the neutron binding energy. The procedure for extracting the total level density $\rho$ from the resonance energy spacing $D$ is described in Ref. [1]. In order to determine the parameter $B$ of Eq. (1.6), which gives the absolute normalization of $\mathscr{T}$, we utilize experimental data on the average total radiative width $\left\langle\Gamma_{\gamma}\right\rangle$ of neutron resonances at $B_{n}$.

The level density is closely connected to the entropy $S$ of the system at a given excitation energy $E$ by

$$
S(E)=k_{\mathrm{B}} \ln \Omega(E),
$$

where the multiplicity $\Omega$ is directly proportional to the level density by $\Omega=\rho / \rho_{0}$. The ground states of even-even nuclei represents a well-ordered system with no thermal excitations and are characterized by zero entropy and temperature. Therefore the normalization denominator is determined to obtain $S=\ln \Omega \sim 0$ in the ground state band region. Figure 3 shows the entropies $S$ of ${ }^{170,171,172} \mathrm{Yb}$ obtained from the $\left({ }^{3} \mathrm{He}, \alpha \gamma\right)$ reaction. The entropies of ${ }^{170} \mathrm{Yb}$ and ${ }^{172} \mathrm{Yb}$ follow each other closely as a function of excitation energy. In particular, in the excitation energy region from the ground state up to $2 \mathrm{MeV}, S(E)$ shows very similar shapes. We interpret the strong increase around $1.5 \mathrm{MeV}$ of excitation energy as the breaking of the first Cooper pair. The next increase, which is much more smeared out, terminates near $2.5 \mathrm{MeV}$ and reveals the beginning of the four quasiparticle regime. Above $2.5 \mathrm{MeV}$ the entropy increases linearly.

Recently, the Oslo method was tested on a simulated $P\left(E, E_{\gamma}\right)$ matrix data set produced by the Prague group with the DICEBOX program. The $\mathscr{T}\left(E_{\gamma}\right)$ and $\rho(E)$ functions were extracted in Oslo 

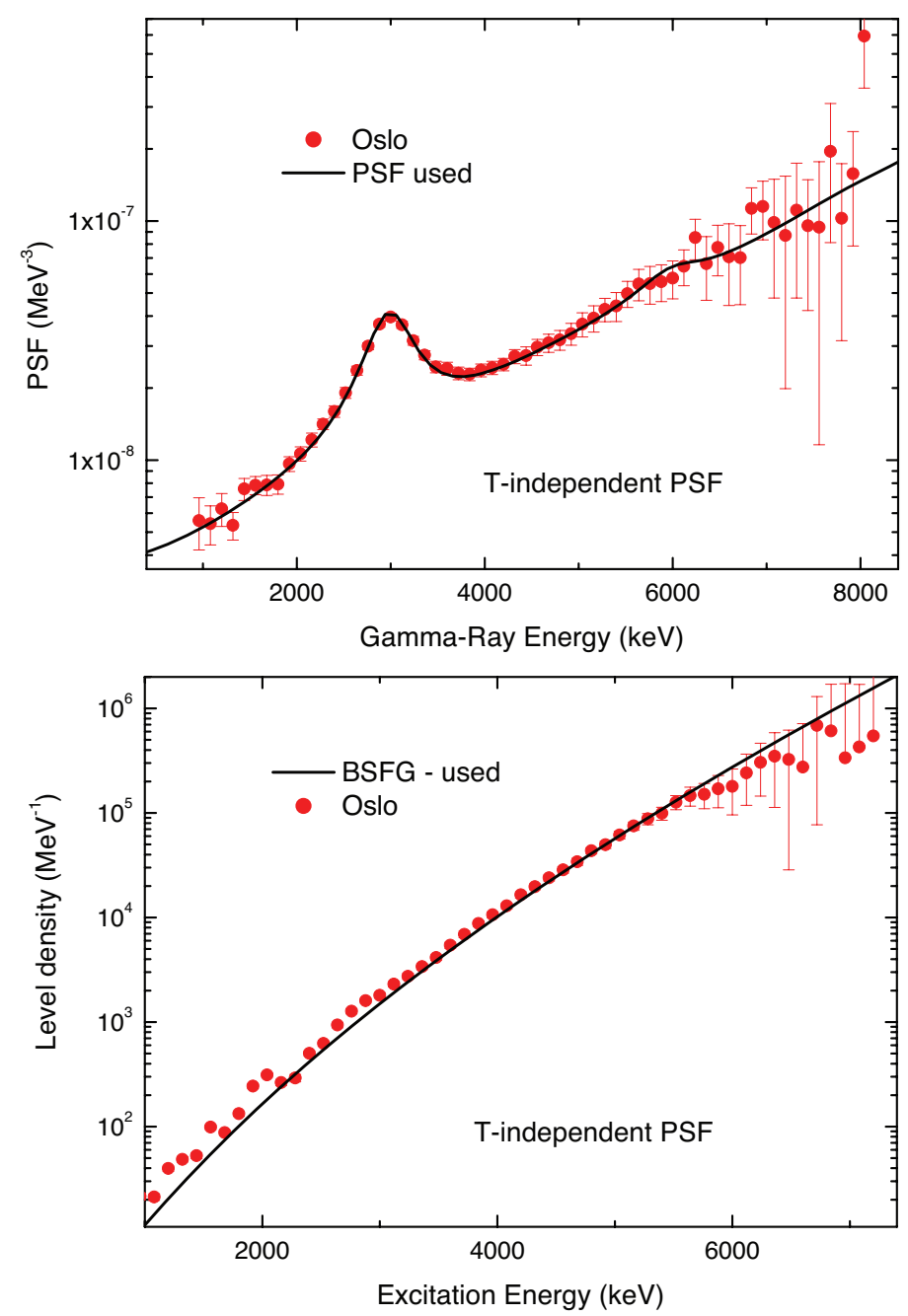

Figure 4: Blind test of the Oslo Method. The solid lines were the input to DICEBOX and the data points were extracted from the simulated $P$ matrix.

and turned out to fit exactly the input functions used by the Prague group. The results are very gratifying and are shown in Fig.4.

In the following we will focus on the photon transmission coefficient $\mathscr{T}\left(E_{\gamma}\right)$, which is directly connected to the PSF.

\section{Gamma decay in continuum}

By assuming only $E 1$ and $M 1$ transitions in the continuum, we may transfer the photon transmission coefficient of Eq. (1.4) into a PSF by

$$
f\left(E_{\gamma}\right)=\frac{1}{2 \pi} \frac{\mathscr{T}\left(E_{\gamma}\right)}{E_{\gamma}^{3}} .
$$

In this way, the PSF can be explored between low $\gamma$-ray energies up to $E_{\gamma} \sim B_{n}-1 \mathrm{MeV}$. 


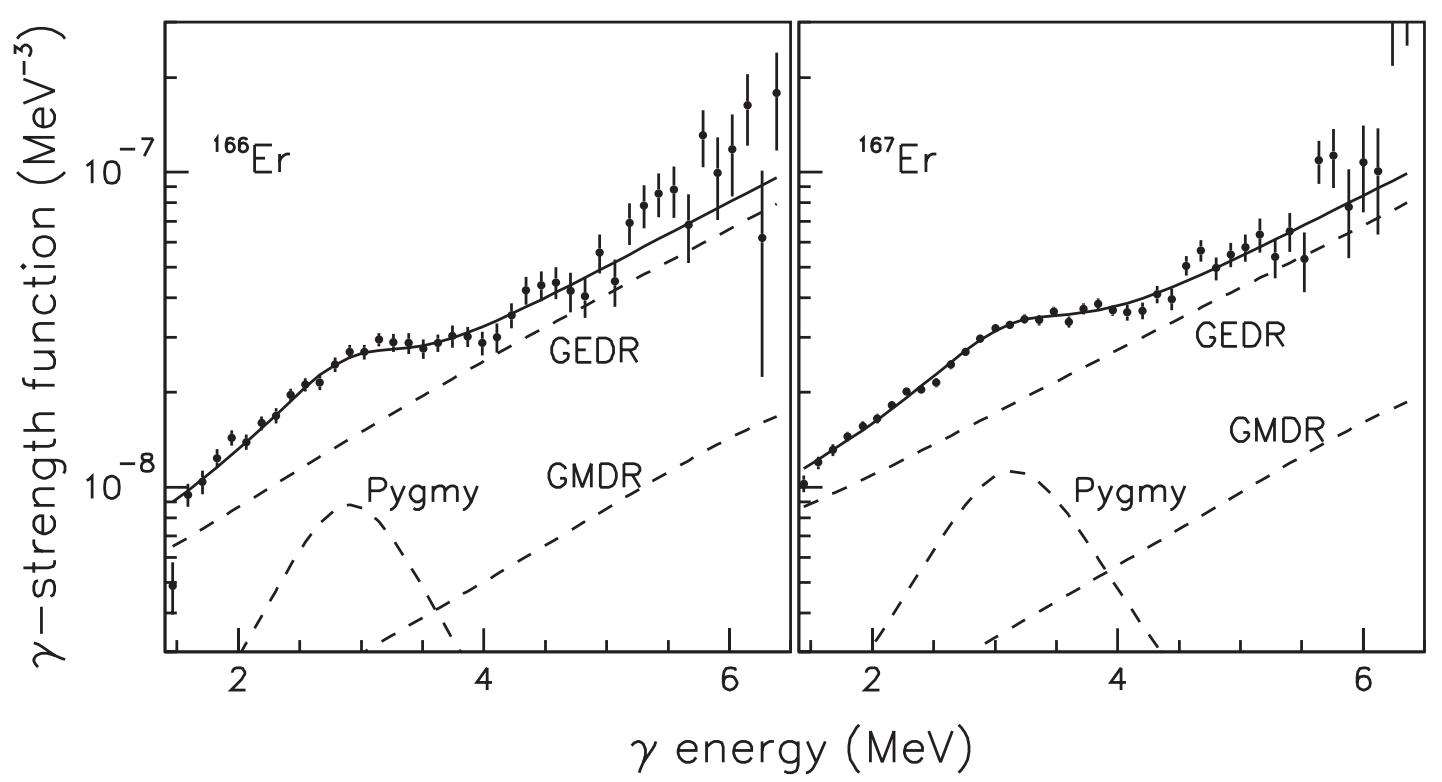

Figure 5: The experimental $\gamma$-ray strength function (data points) of ${ }^{166} \mathrm{Er}$ (left) and ${ }^{167} \mathrm{Er}$ (right). The solid line is the fit to the data by the theoretical model. The dashed lines are the respective contributions of the GEDR, the GMDR, and the pygmy resonance to the total theoretical strength function.

In Fig. 5, the experimental PSF is fitted by a model strength function taking into account both the giant electric dipole resonance and the spin-flip resonance. In addition, a weaker resonance at lower energies is needed in order to fit the experimental data. Because of the much lower strength compared to the GEDR, the resonance is denoted the pygmy resonance. To account for the $E 1$ radiation, the KMF model [9]

$$
f_{\mathrm{E} 1}\left(E_{\gamma}\right)=\frac{1}{3 \pi^{2} \hbar^{2} c^{2}} \frac{0.7 \sigma_{\mathrm{E} 1} \Gamma_{\mathrm{E} 1}^{2}\left(E_{\gamma}^{2}+4 \pi^{2} T^{2}\right)}{E_{\mathrm{E} 1}\left(E_{\gamma}^{2}-E_{\mathrm{E} 1}^{2}\right)^{2}}
$$

is adopted with giant electric dipole resonance parameters $\sigma_{\mathrm{E} 1}, \Gamma_{\mathrm{E} 1}$ and $E_{\mathrm{E} 1}$. The temperature parameter $T$ is utilized as a constant fit parameter. The $M 1$ radiation is described by

$$
f_{\mathrm{M} 1}\left(E_{\gamma}\right)=\frac{1}{3 \pi^{2} \hbar^{2} c^{2}} \frac{\sigma_{\mathrm{M} 1} E_{\gamma} \Gamma_{\mathrm{M} 1}^{2}}{\left(E_{\gamma}^{2}-E_{\mathrm{M} 1}^{2}\right)^{2}+E_{\gamma}^{2} \Gamma_{\mathrm{M} 1}^{2}}
$$

where $\sigma_{\mathrm{M} 1}, \Gamma_{\mathrm{M} 1}$ and $E_{\mathrm{M} 1}$ are the giant magnetic dipole resonance parameters. The pygmy resonance is here described with a similar Lorentzian function $f_{\text {py }}$ as in Eq. (2.3), where the pygmyresonance strength $\sigma_{\mathrm{py}}$, width $\Gamma_{\mathrm{py}}$ and centroid $E_{\mathrm{py}}$ have been fitted in order to adjust the total theoretical strength function

$$
f=\kappa\left(f_{\mathrm{E} 1}+f_{\mathrm{M} 1}\right)+f_{\mathrm{py}}
$$

to the experimental data. The resulting theoretical PSFs are shown as solid lines in Fig. 5 and more details are found in Ref. [5].

Recently [10], these types of low energy pygmy resonances where found to be of $M 1$ scissors mode. Two-step-cascade spectra in ${ }^{172} \mathrm{Yb}$ were measured after thermal neutron capture. They were then compared to calculations based on experimental values of the PSF obtained from the 


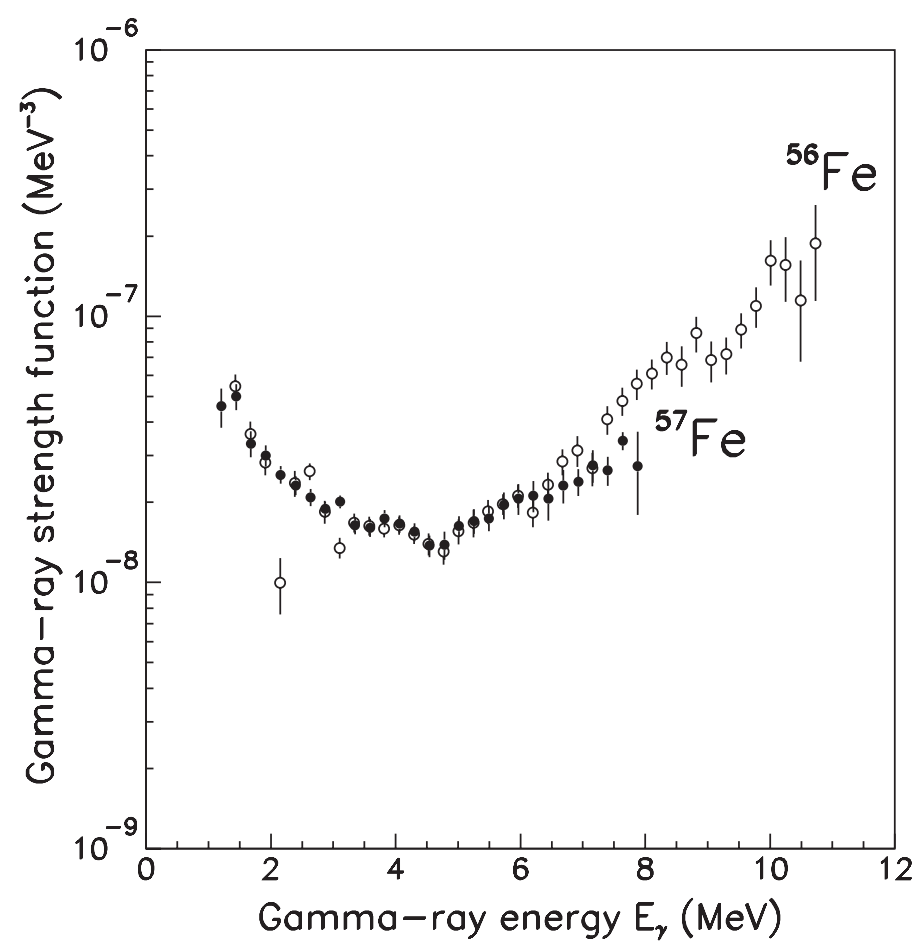

Figure 6: Preliminary photon strength functions for ${ }^{56,57} \mathrm{Fe}$.

${ }^{173} \mathrm{Yb}\left({ }^{3} \mathrm{He}, \alpha \gamma\right){ }^{172} \mathrm{Yb}$ reaction. The centroid of the resonance was found at $E_{\gamma}=3.3(1) \mathrm{MeV}$. The multipolarity was determined to be of $M 1$ type with a strength of $6.5(15) \mu_{\mathrm{N}}^{2}$.

For several lighter nuclei with mass number $A<100$, we find that the PSF is enhanced at low $\gamma$ energies ( $\gamma$ upbend) compared to conventional models. In Fig. 6, this feature is shown for the ${ }^{56,57} \mathrm{Fe}$ nuclei. The data shown here are reanalyzed data compared to the previously published data of Ref. [11].

It has been speculated if the $\gamma$ upbend is due to temperature dependency not included in the extraction procedure. It is well known that the factorization of the Oslo method, see Eq. (1.4) rests on the assumption of constant temperatures for the excitation region studied. In order to check this dependency, we use the rich data set of the ${ }^{96} \mathrm{Mo}\left({ }^{3} \mathrm{He}^{3} \mathrm{He}^{\prime} \gamma\right){ }^{96} \mathrm{Mo}$ reaction, which allows to use the Oslo-method on three statistical independent excitation regions. Within the statistical fluctuations, Fig. 7 shows that all initial excitation energies give qualitatively the same $\gamma$ upbend. Probably, the average temperatures of the final excitation energies for the excitation bins are relatively constant.

\section{Challenges}

There remain several unresolved topics concerning the photon strength function (PSF) of nuclei, which we would like to comment on.

The Oslo method depends crucially on the normalization procedure. In particular, it is important to find the slope of the PSF, determined through the $\alpha$ parameter in Eq. (1.6). In practice, this means to pin-down the level density at $B_{n}$ from the neutron resonance spacing $D$. The transformation from $D$ to $\rho$ requires that the spin cut-off parameter is known. The advice is to use the rigid 


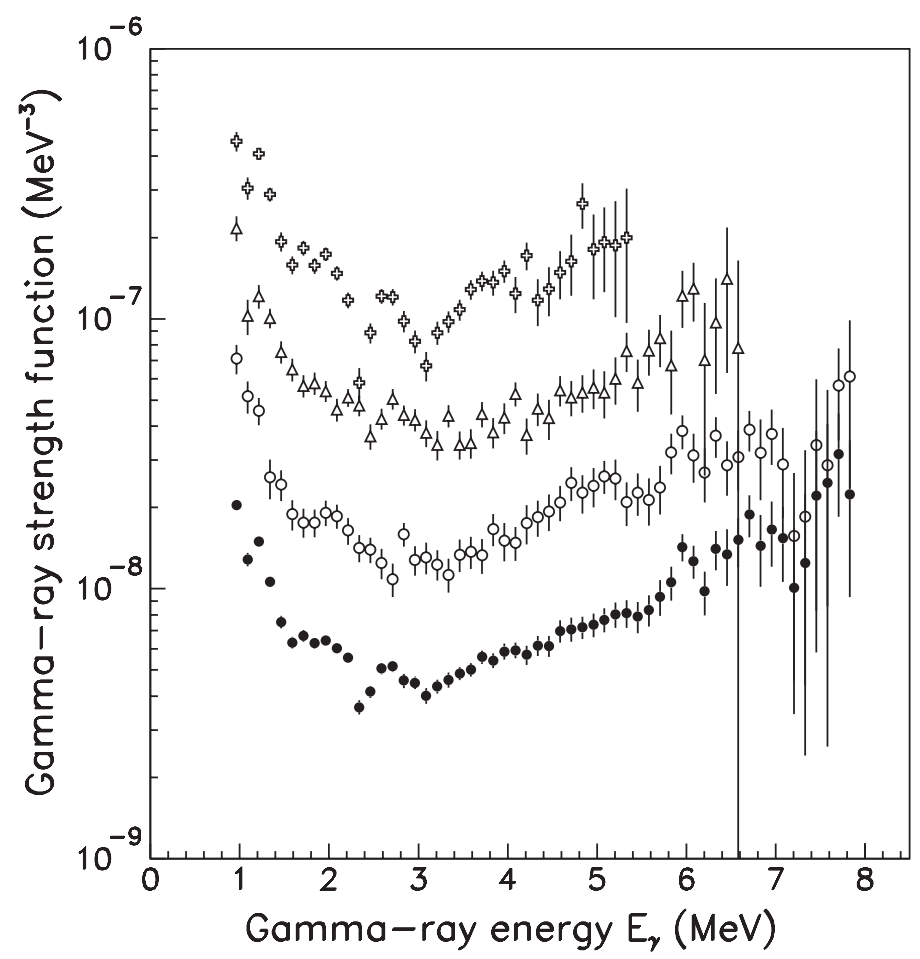

Figure 7: Preliminary photon strength functions for ${ }^{96} \mathrm{Mo}$ using the ${ }^{96} \mathrm{Mo}\left({ }^{3} \mathrm{He},{ }^{3} \mathrm{He} \gamma\right){ }^{96} \mathrm{Mo}$ reaction. Various excitation parts of the $P$ matrix have been used to extract independently PSFs. The excitation regions are (from down and upwards): 4.0-7.9 MeV, 4.0-5.3 MeV, 5.3-6.6 MeV, 6.6-7.9 MeV. In order to separate the four curves, they have been multiplied with 1,3, 9 and 27 , respectively.

moment of inertia in the evaluation of the spin distribution. In some cases the spacing $D$ is unknown, however, the new semi-empirical formulas of Ref. [12] give relatively good extrapolation from one isotope to the next.

There seems also to be inconsistency between the $M 1$ scissor mode strength measured in $\left(\gamma, \gamma^{\prime}\right)$ experiments compared to results from the Oslo method and two-step cascade (TSC) measurements. Probably, the by a factor of two lower strength for the $\left(\gamma, \gamma^{\prime}\right)$ cannot be explained by technical or methodical problems. However, there may very well be different physics in these two cases. The moment of inertia, and thereby the $B(M 1)$ strength, differs if one excite the scissors mode from the ground state or at high excitation energies in continuum. This explanation is a challenge for theoretical investigations.

The PSF should be continuous in the $E_{\gamma} \sim B_{n}$ region, where different techniques are used below and above $B_{n}$. The modeling of the extrapolation of the GEDR tail causes here a substantial problem. Since, up till now, very little is known about the behavior of the PSF below $B_{n}$, there exists an arsenal of different descriptions. The Oslo group has good experience with the KMF model [9], however, how to connect the KMF PSF with the GEDR Lorentzian is still a problem. As if this was not enough, the existence of a possible neutron $E 1$ skin oscillation resonance in this very region obscures the picture. Here, the PSF community should focus on new experiments to extend the systematical data.

As going to lighter nuclei, typically $A<50$, photoabsorption cross section data usually do 
not follow the simple GEDR Lorentzian shape. Preliminary Oslo data in the Sc, Ti and V region indicate that the PSFs contain only minor collective contributions below $B_{n}$. Thus, one should also keep in mind that the concept of PSF at low excitation energy in these nuclei might be meaningless.

The Oslo group has observed enhanced PSF at low $\gamma$-energies in lighter nuclei ( $\mathrm{Si}, \mathrm{Sc}, \mathrm{Ti}, \mathrm{V}$ and Mo). After several years of study, we have not found any experimental, technical or methodical reasons for this unexpected behavior. The effect, called $\gamma$ upbend, is believed to represent new physics going beyond the KMF model. The Norwegian research council (NFR) has initiated a four-year project to further investigate this very interesting phenomenon.

The width of the $M 1$ pygmy resonance seems to disagree in TSC measurements and ${ }^{3} \mathrm{He}-$ induced reactions (Oslo method). The spin transfer of the neutron induced TSC reaction is small, and therefore we plan to use the $\left(p, p^{\prime}\right)$ reaction to see if the $M 1$ pygmy becomes more narrow than measured with ${ }^{3} \mathrm{He}$-induced reactions.

Previous $\left(p, p^{\prime}\right)$ reactions in Oslo gave so high cross section that TSC can be analyzed at all excitation energies up to $B_{n}-1 \mathrm{MeV}$. The Oslo group will focus on the technique to explore the whole TSC matrix, called complete TSC. With the planned implementation of state-of-the-art particle and $\gamma$ detectors, we hope to play an important role in the future of this fascinating field of nuclear physics.

\section{References}

[1] A. Schiller, L. Bergholt, M. Guttormsen, E. Melby, J. Rekstad, and S. Siem, Nucl. Instrum. Methods Phys. Res. A 447, 498 (2000).

[2] M. Guttormsen, T.S. Tveter, L. Bergholt, F. Ingebretsen, and J. Rekstad, Nucl. Instrum. Methods Phys. Res. A 374, 371 (1996).

[3] M. Guttormsen, T. Ramsøy, and J. Rekstad, Nucl. Instrum. Methods Phys. Res. A 255, 518 (1987).

[4] R. Chankova, A. Schiller, U. Agvaanluvsan, E. Algin, L.A. Bernstein, M. Guttormsen, F. Ingebretsen, T. Loennroth, S. Messelt, G.E. Mitchell, J. Rekstad, S. Siem, A.C. Larsen, A. Voinov, S. Odegaard, Phys. Rev. C 73, 034311 (2006).

[5] E. Melby, M. Guttormsen, J. Rekstad, A. Schiller, S. Siem, A. Voinov, Phys. Rev. C 63, 044309 (2001).

[6] P. Axel, Phys. Rev. 126, 671 (1962).

[7] D.M. Brink, Ph.D. thesis, Oxford University, 1955.

[8] U. Agvaanluvsan, A. Schiller, J.A. Becker, L.A. Bernstein, P.E. Garrett, M. Guttormsen, G.E. Mitchell, J. Rekstad, S. Siem, A. Voinov, W. Younes, Phys. Rev. C 70, 054611 (2004).

[9] S.G. Kadmenskiŭ, V.P. Markushev, and V.I. Furman, Yad. Fiz. 37, 277 (1983) [Sov. J. Nucl. Phys. 37, $165(1983)]$.

[10] A. Schiller, A. Voinov, E. Algin, J.A. Becker, L.A. Bernstein, P.E. Garrett, M. Guttormsen, R.O. Nelson, J. Rekstad, S. Siem, Phys. Lett. B 633, 255 (2006).

[11] A. Voinov, E. Algin, U. Agvaanluvsan, T. Belgya, R. Chankova, M. Guttormsen, G. E. Mitchell, J. Rekstad, A. Schiller, S. Siem, Phys. Rev. Lett. 93, 142504 (2004).

[12] T. Egidy and D. Bucurescu, Phys. Rev. C 72, 044311 (2005) and C 73, 049901 (2006) (E). 